\title{
Isomorphisms of Direct Products of Finite Cyclic Groups
}

\author{
Kenichi Arai \\ Hiroyuki Okazaki \\ Tokyo University of Science \\ Chiba, Japan \\ Shinshu University \\ Nagano, Japan \\ Yasunari Shidama
Shinshu University
Nagano, Japan
}

Summary. In this article, we formalize that every finite cyclic group is isomorphic to a direct product of finite cyclic groups which orders are relative prime. This theorem is closely related to the Chinese Remainder theorem ([18]) and is a useful lemma to prove the basis theorem for finite abelian groups and the fundamental theorem of finite abelian groups. Moreover, we formalize some facts about the product of a finite sequence of abelian groups.

MML identifier: GROUP_14, version: $\underline{8.0 .01 \quad 5.4 .1165}$

The notation and terminology used in this paper are introduced in the following articles: [5], [1], [2], [4], [11], [6], [7], [20], [17], [18], [19], [3], [8], [13], [15], [16], $[12],[23],[21],[10],[22],[14]$, and [9].

Let $G$ be an Abelian add-associative right zeroed right complementable non empty additive loop structure. Note that $\langle G\rangle$ is non empty and Abelian group yielding as a finite sequence.

Let $G, F$ be Abelian add-associative right zeroed right complementable non empty additive loop structures. Note that $\langle G, F\rangle$ is non empty and Abelian group yielding as a finite sequence.

We now state the proposition

(1) Let $X$ be an Abelian group. Then there exists a homomorphism $I$ from $X$ to $\prod\langle X\rangle$ such that $I$ is bijective and for every element $x$ of $X$ holds $I(x)=\langle x\rangle$.

(C) 2012 University of Białystok CC-BY-SA License ver. 3.0 or later ISSN 1426-2630(p), 1898-9934(e) 
Let $G, F$ be non empty Abelian group yielding finite sequences. Note that $G \frown F$ is Abelian group yielding.

One can prove the following propositions:

(2) Let $X, Y$ be Abelian groups. Then there exists a homomorphism $I$ from $X \times Y$ to $\Pi\langle X, Y\rangle$ such that $I$ is bijective and for every element $x$ of $X$ and for every element $y$ of $Y$ holds $I(x, y)=\langle x, y\rangle$.

(3) Let $X, Y$ be sequences of groups. Then there exists a homomorphism $I$ from $\prod X \times \prod Y$ to $\prod\left(X^{\frown} Y\right)$ such that

(i) $I$ is bijective, and

(ii) for every element $x$ of $\prod X$ and for every element $y$ of $\prod Y$ there exist finite sequences $x_{1}, y_{1}$ such that $x=x_{1}$ and $y=y_{1}$ and $I(x, y)=x_{1} \frown y_{1}$.

(4) Let $G, F$ be Abelian groups. Then

(i) for every set $x$ holds $x$ is an element of $\prod\langle G, F\rangle$ iff there exists an element $x_{1}$ of $G$ and there exists an element $x_{2}$ of $F$ such that $x=\left\langle x_{1}\right.$, $\left.x_{2}\right\rangle$,

(ii) for all elements $x, y$ of $\prod\langle G, F\rangle$ and for all elements $x_{1}, y_{1}$ of $G$ and for all elements $x_{2}, y_{2}$ of $F$ such that $x=\left\langle x_{1}, x_{2}\right\rangle$ and $y=\left\langle y_{1}, y_{2}\right\rangle$ holds $x+y=\left\langle x_{1}+y_{1}, x_{2}+y_{2}\right\rangle$,

(iii) ${ }^{0} \prod\langle G, F\rangle=\left\langle 0_{G}, 0_{F}\right\rangle$, and

(iv) for every element $x$ of $\prod\langle G, F\rangle$ and for every element $x_{1}$ of $G$ and for every element $x_{2}$ of $F$ such that $x=\left\langle x_{1}, x_{2}\right\rangle$ holds $-x=\left\langle-x_{1},-x_{2}\right\rangle$.

(5) Let $G, F$ be Abelian groups. Then

(i) for every set $x$ holds $x$ is an element of $G \times F$ iff there exists an element $x_{1}$ of $G$ and there exists an element $x_{2}$ of $F$ such that $x=\left\langle x_{1}, x_{2}\right\rangle$,

(ii) for all elements $x, y$ of $G \times F$ and for all elements $x_{1}, y_{1}$ of $G$ and for all elements $x_{2}, y_{2}$ of $F$ such that $x=\left\langle x_{1}, x_{2}\right\rangle$ and $y=\left\langle y_{1}, y_{2}\right\rangle$ holds $x+y=\left\langle x_{1}+y_{1}, x_{2}+y_{2}\right\rangle$,

(iii) $0_{G \times F}=\left\langle 0_{G}, 0_{F}\right\rangle$, and

(iv) for every element $x$ of $G \times F$ and for every element $x_{1}$ of $G$ and for every element $x_{2}$ of $F$ such that $x=\left\langle x_{1}, x_{2}\right\rangle$ holds $-x=\left\langle-x_{1},-x_{2}\right\rangle$.

(6) Let $G, H, I$ be groups, $h$ be a homomorphism from $G$ to $H$, and $h_{1}$ be a homomorphism from $H$ to $I$. Then $h_{1} \cdot h$ is a homomorphism from $G$ to $I$.

Let $G, H, I$ be groups, let $h$ be a homomorphism from $G$ to $H$, and let $h_{1}$ be a homomorphism from $H$ to $I$. Then $h_{1} \cdot h$ is a homomorphism from $G$ to $I$.

One can prove the following propositions:

(7) Let $G, H$ be groups and $h$ be a homomorphism from $G$ to $H$. If $h$ is bijective, then $h^{-1}$ is a homomorphism from $H$ to $G$.

(8) Let $X, Y$ be sequences of groups. Then there exists a homomorphism $I$ from $\prod\left\langle\prod X, \Pi Y\right\rangle$ to $\prod\left(X^{\frown} Y\right)$ such that

(i) $I$ is bijective, and 
(ii) for every element $x$ of $\prod X$ and for every element $y$ of $\prod Y$ there exist finite sequences $x_{1}, y_{1}$ such that $x=x_{1}$ and $y=y_{1}$ and $\left.I(\langle x, y\rangle)=x_{1}\right\urcorner y_{1}$.

(9) Let $X, Y$ be Abelian groups. Then there exists a homomorphism $I$ from $X \times Y$ to $X \times \prod\langle Y\rangle$ such that $I$ is bijective and for every element $x$ of $X$ and for every element $y$ of $Y$ holds $I(x, y)=\langle x,\langle y\rangle\rangle$.

(10) Let $X$ be a sequence of groups and $Y$ be an Abelian group. Then there exists a homomorphism $I$ from $\prod X \times Y$ to $\prod\left(X^{\frown}\langle Y\rangle\right)$ such that

(i) $I$ is bijective, and

(ii) for every element $x$ of $\prod X$ and for every element $y$ of $Y$ there exist finite sequences $x_{1}, y_{1}$ such that $x=x_{1}$ and $\langle y\rangle=y_{1}$ and $I(x, y)=x_{1} \frown y_{1}$.

(11) Let $n$ be a non zero natural number. Then the additive loop structure of $\left(\mathbb{Z}_{n}^{\mathrm{R}}\right)$ is non empty, Abelian, right complementable, add-associative, and right zeroed.

Let $n$ be a natural number. The functor $\mathbb{Z} / n \mathbb{Z}$ yields an additive loop structure and is defined by:

(Def. 1) $\mathbb{Z} / n \mathbb{Z}=$ the additive loop structure of $\left(\mathbb{Z}_{n}^{\mathrm{R}}\right)$.

Let $n$ be a non zero natural number. Observe that $\mathbb{Z} / n \mathbb{Z}$ is non empty and strict.

Let $n$ be a non zero natural number. Note that $\mathbb{Z} / n \mathbb{Z}$ is Abelian, right complementable, add-associative, and right zeroed.

Next we state a number of propositions:

(12) Let $X$ be a sequence of groups, $x, y, z$ be elements of $\prod X$, and $x_{1}, y_{1}$, $z_{1}$ be finite sequences. Suppose $x=x_{1}$ and $y=y_{1}$ and $z=z_{1}$. Then $z=x+y$ if and only if for every element $j$ of $\operatorname{dom} \bar{X}$ holds $z_{1}(j)=($ the addition of $X(j))\left(x_{1}(j), y_{1}(j)\right)$.

(13) For every CR-sequence $m$ and for every natural number $j$ and for every integer $x$ such that $j \in \operatorname{dom} m$ holds $x \bmod \prod m \bmod m(j)=x \bmod m(j)$.

(14) Let $m$ be a CR-sequence and $X$ be a sequence of groups. Suppose len $m=$ len $X$ and for every element $i$ of $\mathbb{N}$ such that $i \in \operatorname{dom} X$ there exists a non zero natural number $m_{1}$ such that $m_{1}=m(i)$ and $X(i)=\mathbb{Z} / m_{1} \mathbb{Z}$. Then there exists a homomorphism $I$ from $\mathbb{Z} /\left(\prod m\right) \mathbb{Z}$ to $\prod X$ such that for every integer $x$ if $x \in$ the carrier of $\mathbb{Z} /\left(\prod m\right) \mathbb{Z}$, then $I(x)=\bmod (x, m)$.

(15) Let $X, Y$ be non empty sets. Then there exists a function $I$ from $X \times$ $Y$ into $X \times \prod\langle Y\rangle$ such that $I$ is one-to-one and onto and for all sets $x, y$ such that $x \in X$ and $y \in Y$ holds $I(x, y)=\langle x,\langle y\rangle\rangle$.

(16) For every non empty set $X$ holds $\overline{\overline{\prod\langle X\rangle}}=\overline{\bar{X}}$.

(17) Let $X$ be a non-empty non empty finite sequence and $Y$ be a non empty set. Then there exists a function $I$ from $\prod X \times Y$ into $\prod\left(X^{\frown}\langle Y\rangle\right)$ such that

(i) $I$ is one-to-one and onto, and 
(ii) for all sets $x, y$ such that $x \in \prod X$ and $y \in Y$ there exist finite sequences $x_{1}, y_{1}$ such that $x=x_{1}$ and $\langle y\rangle=y_{1}$ and $I(x, y)=x_{1} \frown y_{1}$.

(18) Let $m$ be a finite sequence of elements of $\mathbb{N}$ and $X$ be a non-empty non empty finite sequence. Suppose len $m=\operatorname{len} X$ and for every element $i$ of $\mathbb{N}$ such that $i \in \operatorname{dom} X$ holds $\overline{\overline{X(i)}}=m(i)$. Then $\overline{\overline{\prod X}}=\prod m$.

(19) Let $m$ be a CR-sequence and $X$ be a sequence of groups. Suppose len $m=$ len $X$ and for every element $i$ of $\mathbb{N}$ such that $i \in \operatorname{dom} X$ there exists a non zero natural number $m_{1}$ such that $m_{1}=m(i)$ and $X(i)=\mathbb{Z} / m_{1} \mathbb{Z}$. Then the carrier of $\prod X=\prod m$.

(20) Let $m$ be a CR-sequence, $X$ be a sequence of groups, and $I$ be a function from $\mathbb{Z} /\left(\prod m\right) \mathbb{Z}$ into $\prod X$. Suppose that

(i) $\quad$ len $m=\operatorname{len} X$,

(ii) for every element $i$ of $\mathbb{N}$ such that $i \in \operatorname{dom} X$ there exists a non zero natural number $m_{1}$ such that $m_{1}=m(i)$ and $X(i)=\mathbb{Z} / m_{1} \mathbb{Z}$, and

(iii) for every integer $x$ such that $x \in$ the carrier of $\mathbb{Z} /\left(\prod m\right) \mathbb{Z}$ holds $I(x)=$ $\bmod (x, m)$.

Then $I$ is one-to-one.

(21) Let $m$ be a CR-sequence and $X$ be a sequence of groups. Suppose len $m=$ len $X$ and for every element $i$ of $\mathbb{N}$ such that $i \in \operatorname{dom} X$ there exists a non zero natural number $m_{1}$ such that $m_{1}=m(i)$ and $X(i)=\mathbb{Z} / m_{1} \mathbb{Z}$. Then there exists a homomorphism $I$ from $\mathbb{Z} /\left(\prod m\right) \mathbb{Z}$ to $\prod X$ such that $I$ is bijective and for every integer $x$ such that $x \in$ the carrier of $\mathbb{Z} /\left(\prod m\right) \mathbb{Z}$ holds $I(x)=\bmod (x, m)$.

\section{REFERENCES}

[1] Grzegorz Bancerek. Cardinal numbers. Formalized Mathematics, 1(2):377-382, 1990.

[2] Grzegorz Bancerek. König's theorem. Formalized Mathematics, 1(3):589-593, 1990.

[3] Grzegorz Bancerek. The ordinal numbers. Formalized Mathematics, 1(1):91-96, 1990.

[4] Grzegorz Bancerek and Krzysztof Hryniewiecki. Segments of natural numbers and finite sequences. Formalized Mathematics, 1(1):107-114, 1990.

[5] Czesław Byliński. Binary operations. Formalized Mathematics, 1(1):175-180, 1990.

[6] Czesław Byliński. Functions and their basic properties. Formalized Mathematics, 1(1):5565, 1990.

[7] Czesław Byliński. Functions from a set to a set. Formalized Mathematics, 1(1):153-164, 1990.

[8] Czesław Byliński. Partial functions. Formalized Mathematics, 1(2):357-367, 1990.

[9] Czesław Byliński. Some basic properties of sets. Formalized Mathematics, 1(1):47-53, 1990.

[10] Czesław Byliński. The sum and product of finite sequences of real numbers. Formalized Mathematics, 1(4):661-668, 1990.

[11] Agata Darmochwał. Finite sets. Formalized Mathematics, 1(1):165-167, 1990.

[12] Andrzej Kondracki. Basic properties of rational numbers. Formalized Mathematics, 1(5):841-845, 1990.

[13] Artur Korniłowicz. On the real valued functions. Formalized Mathematics, 13(1):181-187, 2005.

[14] Eugeniusz Kusak, Wojciech Leończuk, and Michał Muzalewski. Abelian groups, fields and vector spaces. Formalized Mathematics, 1(2):335-342, 1990. 
[15] Anna Lango and Grzegorz Bancerek. Product of families of groups and vector spaces. Formalized Mathematics, 3(2):235-240, 1992.

[16] Hiroyuki Okazaki, Noboru Endou, and Yasunari Shidama. Cartesian products of family of real linear spaces. Formalized Mathematics, 19(1):51-59, 2011, doi: 10.2478/v10037011-0009-2

[17] Christoph Schwarzweller. The ring of integers, Euclidean rings and modulo integers. Formalized Mathematics, 8(1):29-34, 1999.

[18] Christoph Schwarzweller. Modular integer arithmetic. Formalized Mathematics, 16(3):247-252, 2008, doi:10.2478/v10037-008-0029-8.

[19] Andrzej Trybulec. On the sets inhabited by numbers. Formalized Mathematics, 11(4):341$347,2003$.

[20] Michał J. Trybulec. Integers. Formalized Mathematics, 1(3):501-505, 1990.

[21] Wojciech A. Trybulec. Vectors in real linear space. Formalized Mathematics, 1(2):291-296, 1990.

[22] Zinaida Trybulec. Properties of subsets. Formalized Mathematics, 1(1):67-71, 1990.

[23] Edmund Woronowicz. Relations and their basic properties. Formalized Mathematics, $1(\mathbf{1}): 73-83,1990$.

Received August 27, 2012 